\title{
Thermodynamics of an Ideal Gas of Bosons Harmonically Trapped: Equation of State and Susceptibilities
}

\author{
Víctor Romero-Rochín ${ }^{1}$ and Vanderlei S. Bagnato ${ }^{2}$ \\ ${ }^{1}$ Instituto de Física, Universidad Nacional Autónoma de México, \\ Apartado Postal 20-364, 01000 México, D.F., Mexico and \\ ${ }^{2}$ Instituto de Física, Universidade de São Paulo, C. Postal 369, 13560-970, São Carlos, SP, Brasil \\ Received on 24 November, 2004
}

\begin{abstract}
We present theoretical aspects concerning the thermodynamics of an ideal bosonic gas trapped by a harmonic potential. Working in the Grand Canonical ensemble we are able to properly identify the extensive thermodynamic variable equivalent to the volume and the intensive thermodynamic variable equivalent to the pressure. These are called the "harmonic volume" and the "harmonic pressure" and their physical meaning is discussed. With these variables, the problem of Bose-Einstein condensation is studied in terms of the behavior of the corresponding equation of state and in terms of measurable susceptibilities such as the heat capacities, the isothermal compressibility and the coefficient of thermal expansion. From the analysis, an interesting analogy with BlackBody radiation emerges, showing that at and below the critical temperature, the non-condensate fraction of atoms behaves thermodynamically like a gas of massless particles.
\end{abstract}

\section{INTRODUCTION}

The achievement of Bose-Einstein condensation (BEC) in dilute atomic gases represents the establishment of several new possibilities. A coherent sample of atoms occupying the same quantum state has promoted the realization of coherent beams of matter waves[1]. The investigation of degenerate quantum gases provides a good testing ground for innumerable many-body theories developed along the last few decades within the context of explaining properties of ${ }^{4} \mathrm{He}$ and ${ }^{3} \mathrm{He}[2]$. The field of BEC in trapped particles has evolved very rapidly exceeding the original expectations. BEC is indeed a new window into the quantum world and new topics related to BEC seem to be a constant in many important journals [3]. When an ideal gas is considered, BEC looks to be a paradigm of quantum statistical mechanics offering profound insights into macroscopic manifestations of quantum phenomena.

Many recently published theoretical papers deal with BEC for a harmonic trapped gas[4-6]. Normally, the main concern of those publications is with respect to the condensate fraction of atoms, in which properties associated with zerotemperature BEC have been extensively explored. In this case, the condensate wave function is the solution of the non-linear Gross-Pitaevskii equation, and elementary excitations, [7] vortex states[8] and coherence associated properties[9] are only a few of the effects studied using different approaches. However, little concern has been devoted to the non-condensate fraction present at finite temperatures.

In the case of finite temperature condensates we have the situation where only part of the atomic cloud is participating in the macroscopic occupation of the ground state, and its properties are the subject of current theoretical and even experimental scrutiny. The main attention has been towards the evaluation of density distributions[10]. In fact, the correct assignment of the two components of the bimodal distribution to the condensate and non-condensate parts is a main experimental advantage of the present alkalies condensates over traditional liquid-helium systems. All the extracted properties from the mixed cloud of atoms are obtained from the analysis of the images containing the density profiles.

An aspect that has not been fully explored concerns the thermodynamics of the BEC in a harmonic trap. Identification of extensive and intensive macroscopic variables (like volume and pressure respectively) and their interconnection in an equation of state has not been fully appreciated. Here we discuss how the extensive mechanical variable, usually played by the volume, is replaced by the cube of the inverse of the frequency of the trap. At the same time, there appears an intensive variable, conjugate to the previous one, that replaces the pressure. The latter variable we call the "harmonic pressure", and we shall show that it is not only the formal analog of the hydrostatic pressure but also has the same physical meaning. That is, we shall see that this variable is the one responsible for the mechanical equilibrium of the fluid; see Refs.[11, 12] for further discussion on this point. The identification of such variables allows an easy analysis for a large variety of properties including heat capacities, and other susceptibilities, and a more traditional view of BEC in a gas trapped by an inhomogeneous potential in terms of isothermal curves.

In this paper we consider an ideal bosonic gas trapped by a harmonic potential. Using the traditional Grand Canonical ensemble, the main thermodynamic properties are obtained. Extensive and intensive variables are properly identified allowing for an analysis of the problem in terms of equations of state and susceptibilities. Investigating the properties of the non-condensate fraction at and below $T_{c}$ (critical temperature) an intriguing behavior is obtained: the non-condensate cloud obeys the same thermodynamics of the well known BlackBody Radiation (BBR) problem. In other words, the noncondensate fraction of the harmonic trapped cloud behaves thermodynamically as a gas of massless particles. This intriguing coincidence may reveal a more fundamental relation between BEC and BBR, which certainly demands more consideration and experimental exploration.

We start the paper providing a textbook-like treatment for a gas of bosons confined in a harmonic trap, with details given in Section II to IV. Although there are already in the literature 
many discussions on the thermodynamics of these systems, see Ref.[13] for instance, the approach through the proper mechanical variables here discussed and used has not been fully done before. This article fills such a gap. The analysis is then followed in Section $\mathrm{V}$ by the establishment of an analogy between the non-condensate atoms at and below $T_{c}$ with the Black-Body radiation system. Finally, conclusions and possible applications of these results are given.

\section{THERMODYNAMICS OF AN IDEAL GAS OF BOSONS} IN A HARMONIC TRAP

An important feature associated with available traps of atoms is that the confining potential can be safely approximated by a harmonic-type dependence. Thus, taking harmonic traps as a good approach to reality, we start by considering $N$ bosons of mass $m$ in a 3D isotropic harmonic trap, characterized by the Hamiltonian

$$
\hat{H}_{N}=\sum_{i=1}^{N}\left(\frac{\vec{p}_{i}^{2}}{2 m}+\frac{1}{2} m \omega^{2} \vec{r}^{2}\right)
$$

or, in terms of the number operators $\widehat{n}$,

$$
\hat{H}_{N}=\sum_{i=1}^{N} \hbar \omega\left(\hat{n}_{i}^{x}+\hat{n}_{i}^{y}+\hat{n}_{i}^{z}\right)
$$

with the zero-point energy equal to zero.

It is straightforward to show[14] that in this case the Grand Potential in the Grand Canonical ensemble is given by $(\alpha=$ $\mu / k T)$

$$
\Omega(\mu, T, \omega)=k T \sum_{i j k} \ln \left(1-e^{-\beta \hbar \omega(i+j+k)+\alpha}\right)
$$

where $i, j, k$ are integers that run from 0 to $\infty$. By noticing that the energy is $\varepsilon=\hbar \omega(i+j=k)$, we can perform the sums by introducing a continuous density of states $\rho(\varepsilon) d \varepsilon$. For a 3D harmonic oscillator the density of states is $\rho(\varepsilon)=\varepsilon^{2} /(\hbar \omega)^{3}$. Thus, the Grand Potential can be written as an integration resulting in

$$
\Omega(\mu, T, \omega)=-\frac{k T}{6} \frac{1}{(\beta \hbar \omega)^{3}} \int_{0}^{\infty} d x \frac{x^{3}}{e^{x-\alpha}-1}
$$

where, as usual, the zero-point energy contribution arising from $i=j=k=0$ has been excluded.

Introducing the Bose function,

$$
g_{n}(z)=\frac{1}{\Gamma(n)} \int_{0}^{\infty} d x \frac{x^{n-1}}{z^{-1} e^{x}-1}
$$

where $z=\exp (\alpha)$ is the fugacity, we can therefore write,

$$
\Omega(\mu, T, \omega)=-k T\left(\frac{k T}{\hbar \omega}\right)^{3} g_{4}(z) .
$$

Following the standard procedure, the average number of particles $N$, the average internal energy $E$ and the entropy $S$, as functions of $\mu, T, \omega$, can be obtained:

$$
\begin{gathered}
N=\left(\frac{k T}{\hbar \omega}\right)^{3} g_{3}(z), \\
E=3 k T\left(\frac{k T}{\hbar \omega}\right)^{3} g_{4}(z)
\end{gathered}
$$

and

$$
S=k\left(\frac{k T}{\hbar \omega}\right)^{3}\left[4 g_{4}(z)-\alpha g_{3}(z)\right] .
$$

These are the same formulae found by using a different approach. [4] The interesting aspect we want to point out is that, following standard thermodynamics, the right-hand-side of $\Omega$ in Eq.(6), must be equal to (minus) the product of an intensive thermodynamic variable times an extensive thermodynamic variable. The former being the analog of the pressure, while the latter equivalent to the volume. The physical interpretation of those variable must be done with special care, since the inhomogeneous potential does not allow for an simple characterization of pressure and volume.

First, its clear that the frequency $\omega$ of the trap is a thermodynamic variable. Imagine an adiabatic "compression" or "expansion" of the trap at constant $N$, namely, a change of the frequency of the trap without allowing for change of particles. From Eqs.(7) and (9) we see that the system will heat up or cool down. Further, also from Eqs.(7)-(9), we find that $\omega^{-3}$ must be an extensive variable: $N$ is by definition extensive; therefore, if $N$ is changed to $\lambda N$ with $\lambda>0$, for $T$ and $\mu$ fixed, then, in order for $E$ and $S$ to be extensive they must also change to $\lambda E$ and $\lambda S$. This can only be true if and only if $\omega^{-3} \rightarrow \lambda \omega^{-3}$. Thus, $\omega^{-3}$ is extensive. Let us call it the "harmonic volume" $\mathcal{V}$,

$$
\mathcal{V}=\frac{1}{\omega^{3}}
$$

Since the Grand Potential $\Omega$ must also be extensive, then, the conjugate variable to $\mathcal{V}$ must be,

$$
\mathcal{P}=-\left(\frac{\partial \Omega}{\partial \mathcal{V}}\right)_{T, \mu},
$$

and from the expression for the Grand potential (6), this manifestly intensive variable is

$$
\mathcal{P}=k T\left(\frac{k T}{\hbar}\right)^{3} g_{4}(z) .
$$

We shall call this quantity the "harmonic pressure". Note that in full analogy with the usual hydrostatic pressure and usual volume[14] that $\Omega=-\mathcal{P} \mathcal{V}$ and, therefore the First Law of Thermodynamics for these systems is

$$
d E=T d S-\mathcal{P} d \mathcal{V}+\mu d N
$$


That the quantities $\mathcal{V}$ and $\mathcal{P}$ do not have the units of volume and pressure is irrelevant. Their product, however, has the correct units of energy, as with any pair of conjugate variables.

The harmonic volume $\mathcal{V}$ is certainly related to the spatial extension of the system as we now discuss. First, we recall that the particle density of the gas in the trap is given by[4]

$$
\rho(r)=\frac{1}{\lambda_{T}^{3 / 2}} g_{3 / 2}\left(z e^{-\beta V_{\text {trap }}(r)}\right),
$$

where $V_{\text {trap }}(r)=(1 / 2) m \omega^{2} r^{2}$ is the potential energy of the trap and $\lambda_{T}$ is the thermal de Broglie wavelength,

$$
\lambda_{T}=\frac{h}{(2 \pi m k T)^{1 / 2}} .
$$

It is easy to see from Eq.(14) that the spatial extension of this cloud is contained within a volume $\left(k T / m \omega^{2}\right)^{3 / 2}$. That is, the actual volume of the gas scales as $\omega^{-3}$. Notice that since this spatial volume also depends on the temperature, it cannot be an independent thermodynamic variable.

Let us now analyze the physical meaning of the harmonic pressure $\mathcal{P}$. By writing the Grand Potential in the form,

$$
\Omega=-k T \ln \operatorname{Tr} e^{\alpha \hat{N}-\beta \hat{H}_{N}}
$$

with $H_{N}$ given by Eq.(1), and making use of the definition of the harmonic pressure, Eq.(11), it follows straightforwardly that

$$
\begin{aligned}
\mathcal{P} & =\frac{2}{3} \omega^{3}\left\langle\sum_{i=1}^{N} V_{\text {trap }}\left(r_{i}\right)\right\rangle \\
& =\frac{2}{3} \omega^{3} \int d^{3} r \rho(r) \frac{1}{2} m \omega^{2} r^{2},
\end{aligned}
$$

where in the first line the average is taken in the Grand Canonical Ensemble, and in the second line we used Eq.(14). Of course, explicit evaluation of Eq.(17) yields the expression Eq.(12). We now stress out the fact that, because the force exerted by the trap is felt everywhere by the gas, the latter is no longer uniform. That is, the particle density $\rho_{t h}(r)$ is not constant everywhere. This has as a consequence that the pressure is neither uniform. Instead, the stresses that the fluid exerts on itself is given by a pressure tensor $\tilde{P}(r)$ that is neither constant nor isotropic. Since the fluid is in mechanical equilibrium, it must therefore be obeyed that[16]

$$
\nabla \cdot \tilde{P}(r)=\vec{f}(\vec{r})
$$

where $\nabla \cdot \tilde{P}(r)$ is (minus) the force per unit volume that the fluid exerts on the volume $d^{3} r$ around $\vec{r}$ and $\vec{f}(r)$ is the force per unit volume exerted by the trap on the same spatial point,

$$
\vec{f}(\vec{r})=-\rho(r) m \omega^{2} \vec{r}
$$

By taking the scalar product of Eq.(18) with $\vec{r}$ and integrating over all space, we obtain

$$
\int d^{3} r(\nabla \cdot \tilde{P}(r)) \cdot \vec{r}=\int d^{3} r \vec{f}(r) \cdot \vec{r}
$$

Combining Eqs.(17), (19) and (20), we obtain,

$$
\begin{aligned}
\mathcal{P} & =-\frac{1}{3} \omega^{3} \int d^{3} r(\nabla \cdot \tilde{P}(r)) \cdot \vec{r} \\
& =\omega^{3} \int d^{3} r \frac{1}{3} \operatorname{Tr} \tilde{P}(r)
\end{aligned}
$$

where in the second line an integration by parts was performed. The physical meaning of the harmonic pressure $\mathcal{P}$ is clearer now. The first line of Eq.(21) tell us that the harmonic pressure is the total virial of the internal force and from the second line we find that it is the (invariant) trace of the pressure tensor. But these are precisely the "definitions" of "pressure", see Ref.[16], in other words, $\mathcal{P}$ is proportional to the force (per area) that the fluid exerts on itself and against the trap in order to maintain mechanical equilibrium. Notice that if the gas were confined by rigid walls of volume $V$, the hydrostatic pressure is given by $p=(1 / 3) \operatorname{Tr} \tilde{P}$, obeying,

$$
p=V^{-1} \int d^{3} r \frac{1}{3} \operatorname{Tr} \tilde{P},
$$

in full analogy with Eq.(21). Thus, save for units, the hydrostatic pressure and the harmonic pressure have the same physical meaning. From the point of view of Thermodynamics, those are the intensive variables responsible for mechanical equilibrium in the fluid.

Once we have all thermodynamic variables $S, N, \mathcal{V}$ and their conjugates $T, \mu, \mathcal{P}$, we can proceed to find the rest of the thermodynamic properties. In this article, we have chosen to calculate the equations of state, the isotherms in the $\mathcal{P}-\mathcal{V}$ plane and the susceptibilities since those quantities can be measured in current experimental setups.

\section{EQUATION OF STATE}

From the expressions for the harmonic pressure $\mathcal{P}$ and the number of particles $N$, Eqs.(24) and (7) respectively, we can calculate the equation of state $\mathcal{P}=\mathcal{P}(N / \mathcal{V}, T)$ and provide the isotherms $P-\mathcal{V}$ and the isochores $P-T$ at constant number of particles. These are shown in Figs. 1 and 2. Note that the qualitative structure of the equation of state is equal to the usual case of free bosons discussed in many textbook[14]. That is, for large volumes (i.e. low densities), the isotherms approach those of an ideal classical gas; as the volume is lowered Bose-Einstein condensation sets in at a critical value given by the curve $\mathcal{P} \mathcal{V}^{4 / 3}=$ const as shown in Fig. 1 . Below the critical value, only the non-condensate fraction of atoms exert pressure but this remains constant all the way to zero harmonic volume at constant temperature. On the other hand, for fixed density, the harmonic pressure is reduced as the temperature is lowered until it reaches the transition line $P T^{-4}=$ const, where all the isochores merge, and the pressure vanishes as the temperatures tends to zero, see Fig. 2. The explanation is the same as in usual free-BEC: below the transition, as the harmonic volume is reduced (density increased) the harmonic pressure is only temperature dependent and the excess number of particles condensate into the 


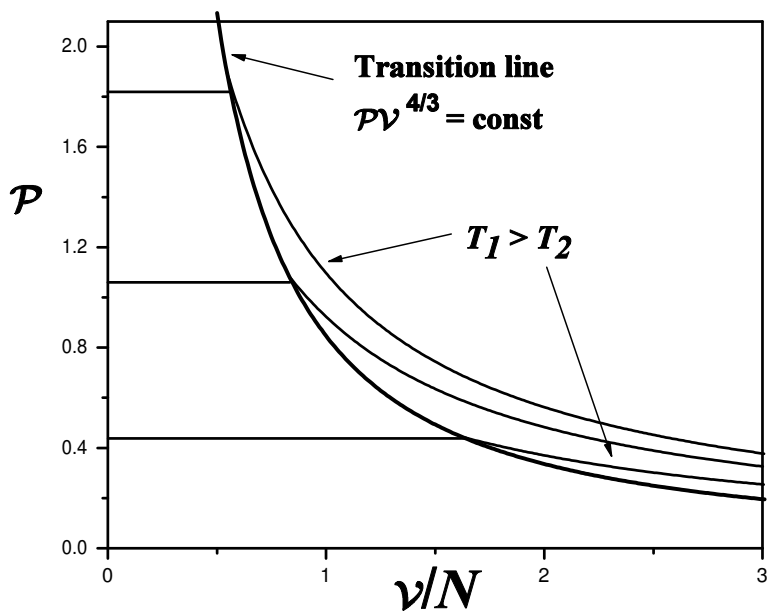

FIG. 1: Isotherms of the $\mathcal{P}(N / \mathcal{V}, T$ equation of state. Below the transition line, the harmonic pressure remains constant. We use units $\hbar=k=1$.

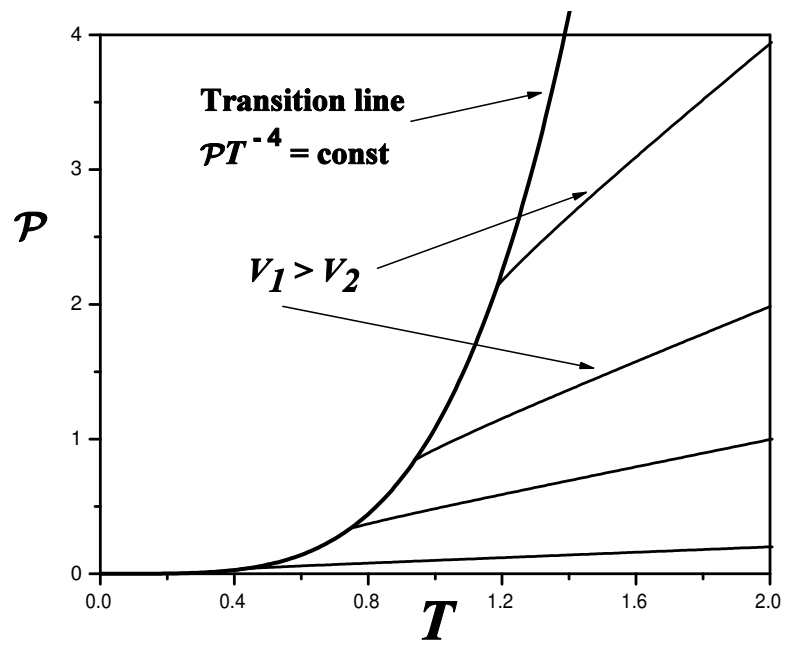

FIG. 2: Isochores of the $\mathcal{P}(N / \mathcal{V}, T$ equation of state. All the isochores merge at the transition line. We use units $\hbar=k=1$.

$i=j=k=0$ ground state. The main difference of our analysis with respect to the free-BEC case is that the in the latter the critical line scale as $p V^{5 / 3}=$ const and $p T^{-5 / 2}$.

From the expressions given in Eqs.(7),(8) and (12) we can deduce further relationships among the harmonic variables and the internal energy. First, we find that the internal energy may be written as

$$
E=3 N k T \frac{g_{4}(z)}{g_{3}(z)}
$$

and the pressure as

$$
\mathcal{P}=\frac{N k T}{\mathcal{V}} \frac{g_{4}(z)}{g_{3}(z)}
$$

Combination of these two yields

$$
E=3 \mathcal{P} \mathcal{V}
$$

Since for high temperatures (i.e. in the classical limit) all the Bose functions become equal, namely $g_{n}(z) \approx \exp (\alpha)$, we find that the equations of state approach those of a classical gas, but of massless or ultrarrelativistic particles. This point will also be left for the next section.

\section{SUSCEPTIBILITIES}

Having identified the harmonic volume and pressure we can now calculate the heat capacities at constant harmonic volume and pressure, $C_{\mathcal{V}}$ and $C_{\mathcal{P}}$; the isothermal compressibility at constant number of particles, $\kappa_{T}$; and the coefficient of thermal expansion $\alpha_{T}$. One must be careful, however, to recall that the formulas given by Eqs.(7)-(9) and (12) are expressed as functions of $T$ and $\mu$ and the susceptibilities are defined at constant number of particles $N$. Moreover, for temperatures equal and lower to the critical temperature, $T \leq T_{c}$, with

$$
T_{c}=\frac{\hbar \omega}{k}\left(\frac{N}{g_{3}(1)}\right)^{1 / 3},
$$

the chemical potential equals zero, $\mu=0$, and, therefore, the number of particles is no longer a thermodynamic variable. This is the phenomenon of BEC in which the thermal properties of the gas are determined by the particles not in the condensate $N_{N C}$ and its number cannot be arbitrarily prescribed because it is already fixed by the temperature and the frequency of the trap, see Eq.(7),

$$
N_{N C}(T)=\left(\frac{\hbar \omega}{k T}\right)^{3} g_{3}(1) .
$$

Therefore, for temperatures above $T_{c}$, the susceptibilities can be calculated directly from Eqs.(7)-(9) and (12) setting $\alpha \neq 0$. For temperatures below $T_{c}$, the entropy depends only on $\omega^{-3}$ and $T$ and the harmonic pressure only depends on $T$ and is independent of $\mathcal{V}$ and $N$ :

$$
S=k\left(\frac{k T}{\hbar \omega}\right)^{3} 4 g_{4}(1) \text { for } T \leq T_{c}
$$

and

$$
\mathcal{P}=k T\left(\frac{k T}{\hbar}\right)^{3} g_{4}(1) \text { for } T \leq T_{c} .
$$

With the above procedure, we find

$$
C_{\mathcal{V}}=3 N k\left[4 \frac{g_{4}(z)}{g_{3}(z)}-3 \alpha \frac{g_{3}(z)}{g_{2}(z)}\right] \text { for } T \geq T_{c}^{+}
$$

and

$$
C_{\mathcal{V}}=12 k V(k T)^{3} g_{4}(1) \text { for } T \leq T_{c}^{-}
$$




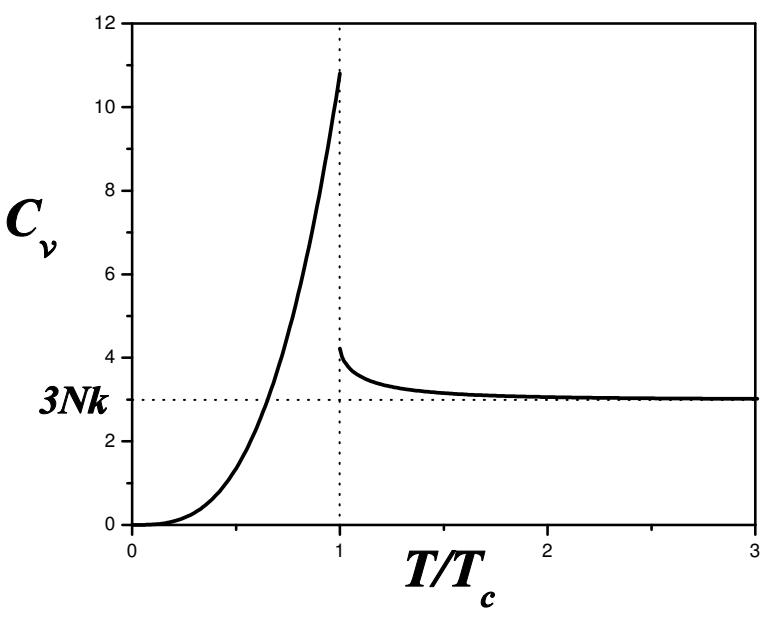

FIG. 3: Heat capacity at constant harmonic volume, $C_{\mathcal{V}}$, as a function of temperature $T / T_{c}$. Note the discontinuity at $T_{c}$ and the classical equipartition value $C_{\mathcal{V}} \rightarrow 3 \mathrm{NK}$ at high temperature.

where $T_{c}^{+}$and $T_{c}^{-}$are the values of $T_{c}$ approached from above and below. The discontinuity at $T_{c}, C_{\mathcal{V}}\left(T_{c}^{-}\right)-C_{\mathcal{V}}\left(T_{c}^{+}\right)$, agrees with the previous calculation of Ref.[4]. The behavior of $C_{\mathcal{V}}$ as a function of temperature is shown in Fig. 3. Similarly,

$$
C_{\mathcal{P}}=4 N k \frac{g_{4}(z) g_{2}(z)}{g_{3}^{2}(z)}\left[4 \frac{g_{4}(z)}{g_{3}(z)}-3 \alpha \frac{g_{3}(z)}{g_{2}(z)}\right] \text { for } T \geq T_{c}^{+} .
$$

For $T \leq T_{c}^{-}$, one finds that $C_{\mathcal{P}}=\infty$. This is not surprising since, as we indicate above, below $T_{c}$ the pressure only depends on temperature, and therefore,

$$
\left(\frac{\partial S}{\partial T}\right)_{P}=\infty \text { for } T \leq T_{c}
$$

That is, if the pressure is kept constant, the temperature cannot be raised no matter how much heat one puts into the system. Note that the ratio $C_{\mathcal{P}} / C_{\mathcal{V}} \rightarrow 4 / 3$ for high temperatures; again, this result is the same of an ultrarrelativistic gas. Fig. 4 shows $C_{P}$.

The isothermal compressibility, defined as $\kappa_{T}=$ $-(1 / \mathcal{V})(\partial \mathcal{V} / \partial \mathcal{P})_{T, N}$, is given by

$$
\kappa_{T}=\frac{V}{N k T} \frac{g_{2}(z)}{g_{3}(z)} \text { for } T \geq T_{c}^{+} .
$$

For $T \leq T_{c}^{-}$, one finds again that $\kappa_{T}=\infty$. The reason for this value comes from the fact that the pressure is independent of the volume for this interval of temperatures (see Fig. 1). For high temperature one finds, as expected, the Curie law-like behavior of an ideal classical gas. Fig. 5 shows $\kappa_{T}$.

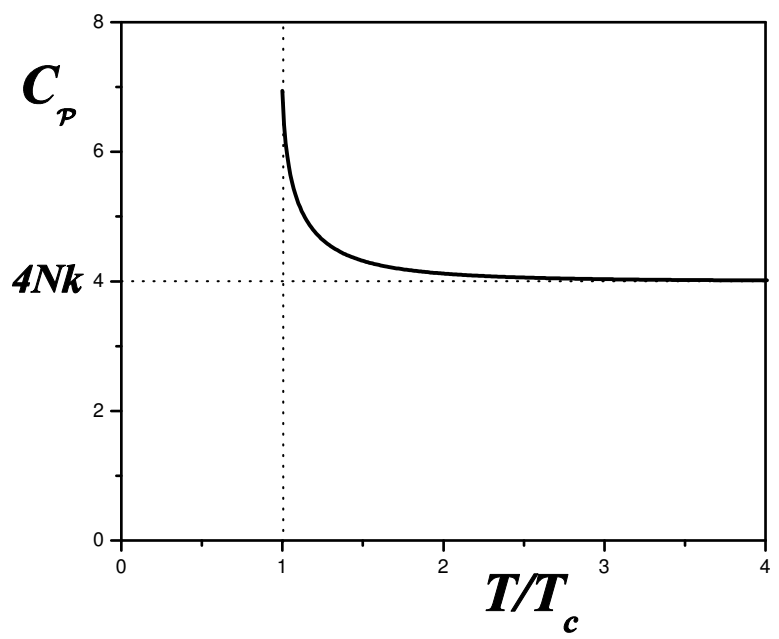

FIG. 4: Heat capacity at constant harmonic pressure, $C_{\mathscr{P}}$, as a function of temperature $T / T_{C}$. Note the discontinuity at $T_{C}$ and the classical value $C_{\mathcal{P}} \rightarrow 4 N K$ at high temperature Below $T_{c}, C_{\mathcal{P}}$ diverges since the harmonic pressure only depends on temperature.

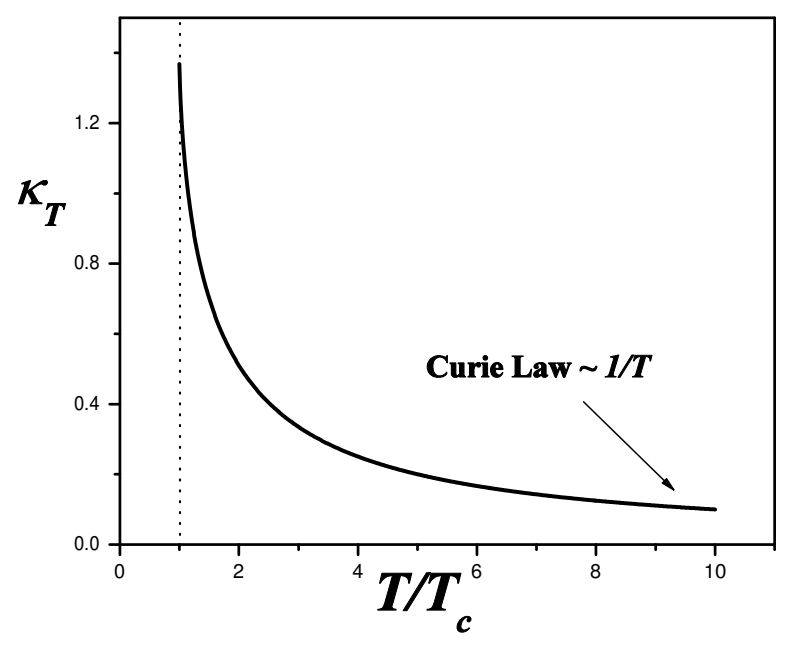

FIG. 5: Isothermal compressibility, $\kappa_{T}$, as a function of temperature $T / T_{C}$. Below $T_{c}, \kappa_{T}$ diverges since the harmonic pressure becomes independent of the harmonic volume, at constant temperature.

For the thermal expansion coefficient, $\alpha_{T}=$ $(1 / \mathcal{V})(\partial \mathcal{V} / \partial T)_{\mathcal{P}, N}$, one gets

$$
\alpha_{T}=\frac{1}{T} \frac{g_{2}(z)}{g_{3}(z)}\left[4 \frac{g_{4}(z)}{g_{3}(z)}-3 \frac{g_{3}(z)}{g_{2}(z)}\right] \text { for } T \geq T_{c}^{+} .
$$

For $T \leq T_{c}^{-}, \alpha_{T}=\infty$, because at fixed pressure, the temperature cannot be changed. Also, for high temperatures $\alpha_{T} \approx 1 / T$, which is the result of an ideal classical case[15]. Fig. 6 shows $\alpha_{T}$.

The fact that the susceptibilities, except $C_{\mathcal{V}}$, become infinite below $T_{c}$ are an indication that the gas not in the condensate behaves in a peculiar way. In the next section, we shall 


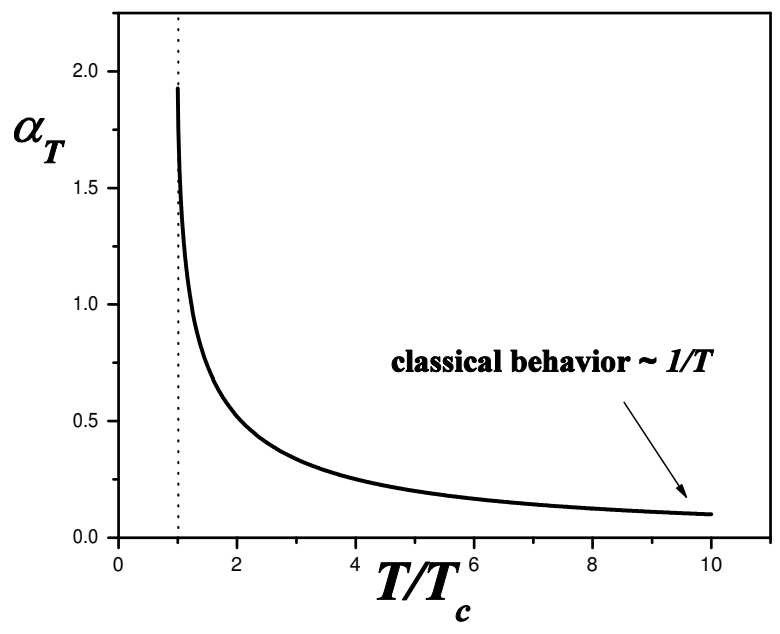

FIG. 6: Coefficient of thermal expansion, $\alpha_{T}$, as a function of temperature $T / T_{c}$. Below $T_{c}, \alpha_{T}$ diverges since temperature becomes independent of the harmonic volume, at constant harmonic pressure.

discuss how these peculiarities are related to the deep analogy of this gas with a gas of photons.

\section{THERMODYNAMIC ANALOGY OF A GAS OF PHOTONS WITH THE NON-CONDENSATE FRACTION OF ATOMS}

In this section we briefly explore the very close and intriguing analogy between the Black-Body radiation problem and the BEC case in a harmonic potential. We shall see that, at and below $T_{c}$, the gas of massive bosons in the non-condensate fraction behaves thermodynamically identically to a gas of photons in thermal equilibrium inside a cavity.

We begin by recalling that the Grand Potential in the BlackBody radiation problem can be cast as,[14]

$$
\Omega_{B B}(T, V)=-16 \pi k T V\left(\frac{k T}{h c}\right)^{3} g_{4}(1)
$$

where $V$ is the actual volume of the cavity. Compare this expression with equation (6) for $\Omega$ of the gas of bosons in the harmonic trap. The latter expression, by arbitrarily multiplying and dividing by $c^{3}$, becomes

$$
\Omega(\mu, T, \omega)=-k T\left(\frac{2 \pi c}{\omega}\right)^{3}\left(\frac{k T}{h c}\right)^{3} g_{4}(z) .
$$

The quantity $(2 \pi c / \omega)^{3}$ now does have units of volume and, by comparing Eq.(37) with Eq.(36) of the free case, we see that indeed it takes the role of the volume. The rest of the terms in Eq.(37) now have units of pressure, and its dependence with $T$ is that of a radiation pressure. Since at and below the BEC transition the chemical potential becomes zero, $\alpha=0$, it follows from the above two equations that the thermodynamics of the photon gas and of the non-condensate fraction of atoms are identical. And as we saw in the previous Section, even some thermodynamic properties in the classical limit resemble those of a gas of ultrarrelativistic particles.

An important reason for this similarity, of course, is the fact that zero chemical potential means that the number of particles is no longer a conserved thermodynamic variable. However, this also happens in the usual free-BEC problem without any identity with BBR. Another reason may be due to the fact that the bosons in the harmonic trap as well as the photons in a cavity can be thought of as harmonic oscillators (and expressed as such in a second-quantization scheme). These two points together may suffice to account for the analogy for temperatures at and below the critical temperature. Although we believe there may be a deeper meaning in the analogy here pointed out, we have only mentioned several interesting aspects about it and we shall leave further analysis to future work. A convenience that comes out from such an analogy is the simple way to obtain all the thermodynamic properties.

\section{CONCLUSIONS}

We have provided a textbook-like approach to evaluate the thermodynamics for a confined boson gas in a harmonic trap. The identification of an extensive variable ("harmonic volume") and an intensive variable ("harmonic pressure") allow to view the problem of BEC in a harmonic potential through the corresponding equation of state, $\mathcal{P}=\mathscr{P}(N / \mathcal{V}, T)$. The corresponding plots (Fig. 1) give a very intuitive general picture of the problem. Important properties such as the susceptibilities and the heat capacities can also be easily evaluated. Since in most of the current experiments what is measured is the the particle density $\rho(\vec{r})$, we see from Eq.(17) that the harmonic pressure can readily be measured, opening the way for an additional type of measurements, and another source of information, of cold gases of alkaline atoms. As a matter of fact, recently this approach was exploited by Magalhães et al. in their achievement of BEC in Brazil[11].

An interesting point not usually mentioned in the current literature is that at and below $T_{c}$ the remaining atoms not in the condensate behave like a massless particle gas presenting all the thermodynamic functions equal to those of Black-Body radiation. One may think as if the non-condensate fraction were a photon-like gas that at $T_{c}$ its particles acquire mass (i.e. when the chemical potential becomes non-zero). Besides being an interesting analogy, this behavior may carry with it a more fundamental explanation.

Finally, it is of interest to mention that, even though, the dependence on temperature and other variables is different in the present problem from the case of a gas of particles contained in a volume $V$, ans this will reflect on quantitative measurable differences, from another perspective, the physics is essentially very similar if the proper thermodynamic variables are elucidated and used. On one hand, we showed in this paper that the physical origin of the harmonic pressure and the "usual" hydrostatic pressure is essentially the same. Here, we would like to discuss another important aspect regarding the role of the de Broglie wavelength: we can see from Eqs.(6), (7)-(9), that the thermal de Broglie wavelength does not ap- 
pear naturally. The thermodynamic quantity that determines whether the system is in the quantum or in the classical regime is now, see Eq.(7),

$$
N\left(\frac{\hbar \omega}{k T}\right)^{3} .
$$

If this quantity is much less than one, the system is classical; quantum effects appear when this quantity is smaller or of the order of one. This formula is to be contrasted with the usual one of a gas of bosons in a vessel of volume $V$,

$$
N \frac{\lambda_{T}^{3}}{V}
$$

with $\lambda_{T}$ the thermal de Broglie wavelength, Eq.(15). From here, we see the very well known fact that the "usual" dependence of the number of particles on the temperature goes as $T^{3 / 2}$ while in the harmonic trap goes as $T^{3}$. However, by introducing the effective volume of the gas in the trap as $V_{e f f}=\left(2 \pi k T / m \omega^{2}\right)^{3 / 2}$, as discussed in Section 2, we can recast the condition of the harmonic trap as

$$
N\left(\frac{\hbar \omega}{k T}\right)^{3}=N \frac{\lambda_{T}^{3}}{V_{e f f}} .
$$

Thus, while the dependence on the temperature is different, the physical meaning remains: quantum effects are important when the de Broglie wavelength is comparable with the average distance among particles $\left(V_{e f f} / N\right)^{1 / 3}$.

\section{Acknowledgments}

Work partially supported by Fapesp (Center for Optics and Photonic Research) and CNPq (Brazil) and by CONACyT (Mexico) through grant 32634-E.
[1] M.R. Andrews, C.G. Townsend, H.J. Miesner, D.S. Dustee, D.M. Kurn, and W. Ketterle, Science 275, 637 (1997).

[2] A.J. Legget, Phys. Scripta T76, 199 (1998).

[3] See K. Burnett, M. Edwards, and C. Clark, Phys. Today 52, 37 (1999).

[4] V.S. Bagnato, D.E. Pritchard, and D. Kleppner, Phys. Rev. A 35, 4354 (1987).

[5] J. Schneider and H. Wallis, Phys. Rev. A 57, 1253 (1998)

[6] F. Daltovo, S. Giorgini, L.P. Pitaevskii, and S. Stringari, Rev. Mod. Phys. 71, 463 (1999).

[7] D.M. Stamper, H.J. Miesner, S. Inowye, M. Andrews, and W. Ketterle, Phys. Rev. Lett. 81, 500 (1998).

[8] K.P. Martin and W. Zhang, Phys. Rev.A 57, 4761 (1998).

[9] W. Ketterle and H.J. Miesner, Phys. Rev. A 56, 3291 (1997).
[10] L.U. Hau, B.B. Busch, C. Liu, Z. Dutton, M.M. Burns, and J.A. Golovchenko, Phys. Rev. A 58, R54 (1998).

[11] K.M.F. Magalhães, S.R. Muniz, E.A.L. Henn, R.R. Silva, L.G. Marcassa, and V.S. Bagnato, Las. Phys. Lett. 2, 214 (2005).

[12] V. Romero-Rochin, Phys. Rev. Lett. 94, 130601 (2005).

[13] C.J. Pethick and H. Smith, Bose-Einstein Condensates in Dilute Gases, (Cambridge University Press, Cambridge, 2002).

[14] R.K. Pathria, Statistical Mechanics, (Pergamon Press, Oxford, 1972).

[15] H.B. Callen, Thermodynamics (J. Wiley and Sons, New York, 1960)

[16] L. Landau and L. Lifshtiz, Fluid Mechanics (Pergamon, Oxford, 1959). 